\title{
Genetic and Clinical Tests: New Strategy for Cardio- Genetic Laboratories in Uzbekistan: Short Review
}

\author{
Aleksandr Nagay*, Kurbanov RD, Khamidullaeva GA, Srojidiniva NZ and Tursunova NB \\ *Department of Arterial Hypertension and Molecular Genetics Research, Republican specialized center of cardiology, Uzbekistan
}

Submission: May 11, 2017; Published: June, 30, 2017

*Corresponding author: Aleksandr Nagay, Department of Arterial Hypertension and Molecular Genetics Research, Republican Specialized Center of Cardiology 4, Osiyo Str Mirzo-Ulugbek 100052, Tashkent Uzbekistan, Tel: +998903503409; Email: alexsan1984@mail.ru

\begin{abstract}
Introduction
The modern medicine needs reliable databases to help laboratories and clinicians to interpret clinical data of DNA test results [1]. Genetic testing undoubtedly is an important part in clinical practice. Such tests can be used first of all at detection of tumors, markers of vessels lesions or to help with a case of impossibility of diagnosis at standard clinical inspection [2]. In this aspect diagnostics of the essential hypertension $(\mathrm{EH})$ as multi factorial phenomenon with the polygenic nature of inheritance is the most perspective in clinical practice. Unprecedented depreciation of the chip-sequencing, made possible to conduct large researches of cardiovascular diseases.
\end{abstract}

These technologies were always followed by 2 main problems

I. Choice of management of a huge array of genomic data

II. Choice of computing methods of the analysis [3].

Despite it, use of genomic results for 10 years became ubiquitous. Technically genetic tests don't differ from any other medical test. Genomic tests as all other clinical tests, are caused by the probabilistic size where a certain path physiological state is present (that is the diagnosis), or will be present (that is, the forecast) [4]. Anyway, genomic or usual tests are used for clinical decision-making in the context of an asymptomatic or pathological condition of the patient.

As a result clinical genomic data have the same purpose as other medical tests: to provide information at diagnosis and treatment of the patient.

However, genetic tests have also some difference from clinical

1. High probability of the unforeseen obtained data

2. Confidentiality of the obtained information (only for family members)

3. The obtained data don't guarantee that the patient understands character of the provided information (for example: this category includes information on future risks of development of pathologies). Today there are some doubts that highly technological genetic tests of SNP won't be more expensive than medical researches [5].

Today the most important difference between genomic tests and clinical - the fact that medical institutions and medical industrial infrastructure believe that genetic and usual medical tests will be very different because of complexity of interpreting results. Such perception happens because of full shortage of infrastructure support for acquisition of resources for the purpose of processing difficult, genomic bases which are necessary for drawing up of electron medical account [6]. Huge shortage of experts in genomic pathology and bioinformatics and also lack of programs of training in the sphere of clinical genomics in medical colleges and institutes remains problematic aspect still [7].

Keywords: Biomarkers; DNA laboratory; Cardio vascular disease; Essential Hypertension, Single Nucleotide Polymorphism; Arterial Blood Pressure; Cardio-genetic laboratories

\section{Cardio-genetic lab plan stages and constructive features}

Today there are 4 principles of successful expansion of DNA lab in cardiologic sections a. Motivation

b. Automation

c. Technology of the effective genes analysis 


\section{d. Financial profit}

Assessment of working process-the first stage of the project is that how DNA lab will fit in work of cardiologic sections. If assessment of requirements finds processes which need be redesigned they have to be installed before introduction. For example, it concerns to coherence between document flow and synchronization of DNA lab structure; (for example: between DNA tests, biochemical tests and clinical tests). That's why the participation of doctors at all development stages of the project is necessary. Key questions at projection of DNA lab concerns that information is provided, when it is presented, and how it is presented to the user. Information from DNA lab can be presented to the clinician automatically or on demand. Whatever were the features of delivery information, quality of information and proof which are its cornerstone are the major factors defining impacts of DNA lab on patient safety. The key moments of such situations is providing the necessary person with the correct information in the necessary format, in due time.

\section{Superfine Phenotype and Molecular Information and their Clinical Context}

To understand pathophysiology, clinical, imaging, functional, molecular, genomic, markers need to be connected, and further linked to outcomes. Such connection presents a formidable challenge to data storage, management and analysis, IT capacity, and accessibility. Innovative statistics will be needed addressing a multiplicity of potential biomarkers, identifying reliable and valid measurements.

\section{Genotype in CVD}

Integration of biological markers for specific disease processes has the potential to identify patients at risk for first cardiovascular events (arterial hypertension, cerebrovascular or coronary heart diseases, cardiac arrhythmia, heart failure, diabetes type II) to allow to target therapies to patients who are most likely to benefit, and to allow better prediction of unwanted side-effects of therapy.

\section{Automation and Biochip Array Technology}

Technological progress in the area of the laboratory equipment, for example development of biochips for biochemical analyzers "Randox Evidence Evolution", sequenators "Sequencing Ion Chef System AB" of the last generation and technology of a liquid biopsy promotes the development of structurally new laboratory systems of information support. The majority of such systems will allow the regular analyst to begin work without -finding instructing by means of use of the intuitive and simple interface. In the future such systems will inevitably increase automation of labor-consuming laboratory office-work.

\section{Technology of the Effective Genes Analysis}

The technology of the effective genes analysis is the combined method of the sequential analysis, ROC-curve and relative risk of RR (Altman's theorem) with use of basic mathematical algorithms (Bayes' theorem) in diagnostics of in vitro. Thus, using DNA identification, the developed algorithm can recommend an individual diet and a safe dose of drug (Warfarin, Klopidogrel, Simvastatin, Perindopril). The algorithm is capable to transform pathogenic genetic data to the diagnostic tool which in a combination with clinical observation and biometric data can create the clinical decision.

\section{The Genetic Test and Financial Profit}

In addition every year government clinics spend appreciable working resources and appliances for laboratory office-work. As a rule, there is completely no integrated DNA tests system in such laboratories. Modern clinical practice shows that, using of the automatic systems increases economic efficiency and effectiveness of laboratory operations. As a result it leads to rapid technical progress of laboratories. As a result for the last 20 years doctors began to rely on constantly extending range of biomedical services, with constantly reduced expenses of working time.

\section{Clinician Motivation}

For the last 25 years, the population of Uzbekistan was enlarged twice. Today such tendency of population increase is followed by its aging. The positive aspect of this phenomenon is augmentation of average life expectancy. However, a negative side that the augmentation of life expectancy doesn't correspond to its quality. Deterioration first of all is bound to augmentation of prevalence of age chronic diseases, such as a hypertension. For example, today in Uzbekistan, one of three adults has raised by the ABP, and excess weight occurs at every second (WHO/STEPS 2014). As a result it caused double increasing of visits of the doctor and total number of patients in general. However, despite a huge load in a health system, the fact that it caused rising of automation of biomedical process was positive. So in Uzbekistan, commercial clinics could enlarge efficiency of office-work due to use of PCR-diagnostics systems in laboratory practice. Over time it allowed to reduce holding time of the patient, to exclude errors of a human factor and will get rid of need to keep big staff. This example confirms efficiency of using DNA technologies as an element of improvement of medical service quality.

\section{Conclusion}

Despite numerous disadvantages of the genomic tests, the analysis of a big flow of genetic and clinical information it became real. We recommend to consider several moments on the first steps. In - the first to pay attention to an analytical stage. To provide sensory management of all knots of system. It is necessary to consider in advance all working platforms which will be served by cardio-genetic lab. Use Bayesian approach to frame a matrix of recognition of the damaging alleles and haplotype. It is necessary to adhere to standard forms of biological data. To strengthen enough genetic information, clinical geneticist has to be able to structure a superfine phenotype. For stratification of risk of rising of the ABP (the high, increased, average risk) use 
multi-sample option. It will lead to specificity increasing while sensitivity will remain invariable. Use heuristic and statistical algorithms; they will be able to identify the damaging genotype, without conflicting to clinical information of the patient. We also recommend to limit access to genetic information. It is bound to the fact that information on existence of the inherited pathologies can affect a work arrangement and receiving insurance. In some countries laws of genetic non-discrimination (US genetic information nondiscrimination act GINA 2008) are adopted.

Despite a technological capability to process and make more exact clinical assessment of big data array, the problem of inaccessibility of resources and mechanisms of exchange of the annotated genetic information remains unresolved. Ability to authentically predict phenotypical results on the basis of a genotype, still remains a DNA lab task of the next generation.

\section{References}

1. Chute G, Ullman-Cullere (2014) Some experiences and opportunities for big data in translational research. Genet Med 15(10): 802-809.
2. Kircher M, Witten D, Jain P, Roak OB, Cooper G, et al. (2014) A general framework for estimating the relative pathogenicity of human genetic variants. Nat. Genet 46(3): 310-315.

3. Rabbani B, Tekin M, Mahdieh N (2014) The promise of whole-exome sequencing in medical genetics. J Hum Genet 59(1): 5-15.

4. Rehm H, Bale S, Bayrak-Toydemir P, Berg JS, Brown KK, et al. (2013) ACMG clinical laboratory standards for next generation sequencing. Genet Med 15(9): 733-747.

5. Vihinen M, Dunnen J, Dalgleish R, Cotton R (2012) Guidelines for establishing locus specific databases. Hum Mutant 33(2): 298-305.

6. Bennetts B (2014) Quality standards for DNA sequence variation databases to improve clinical management under development in Australia. Appl Transl Genom 3(3): 54-57.

7. Kullo I, Haddad R, Prows CA, Holm I, Sanderson SC, et al. (2014) Return of results in the genomic medicine projects of the eMERGE network. Front Genet 5: 50. 